\title{
Preparation of conductive polyaniline solutions for electronic applications
}

\author{
S K DHAWAN and D C TRIVEDI \\ Central Electrochemical Research Institute, Karaikudi 623 006, India \\ MS received 23 December 1988
}

\begin{abstract}
Electrically conductive processible polyaniline films have been synthesised from the electropolymerization of aniline under non-aqueous conditions. The characterization of the polymer was carried out by spectroscopic methods.
\end{abstract}

Keywords. Soluble polyaniline; electrochemical polymerization.

\section{Introduction}

Electrically conductive polymers are attracting considerable attention in order to explore the possibility of using them in rechargeable batteries (Genies et al 1985), photovoltaics devices (Noufi et al 1982), EMI shielding (Bigg et al 1981) and many other applications in the electronics industry. The characterization and processing of conducting polymers are important unsolved problems from the scientific and industrial point of view. The soluble conducting polymers can find applications such as: (i) conducting ink; (ii) conductive adhesives and (iii) EMI shielding. Till now polyaniline has been known only as a green interactable material (Green 1910), the base form of which was assigned the composition,

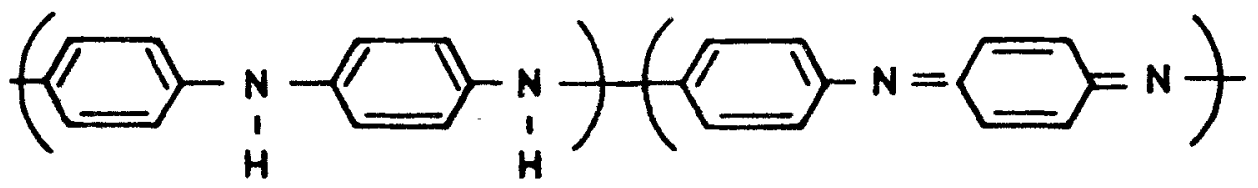

and termed emeraldine, which contains equal numbers of reduced,

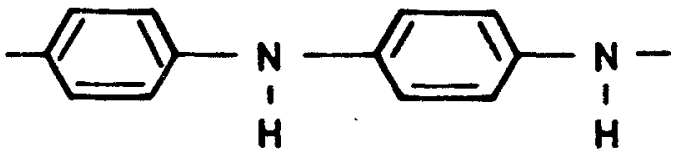

and oxidised units,

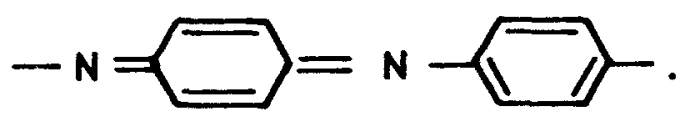

Very little effort has been devoted to the solubilization of this polymer. Soluble polyanilines may be interesting in fundamental studies for evolving physicochemical properties, and for commercial applications as a more easily processible material. Recently soluble polyanilines have been reported (Suzhen Li et al 1987; Dhawan and Trivedi 1988), where it is observed that when polymerization of aniline is carried out with organic proton acids of large molecular size e.g. $p$-toluene sulphonic acid, a soluble polymer of aniline is obtained. Angelopoulos et al (1987) have reported the processible polyaniline from $80 \%$ aqueous acetic acid. But if the same polymerization is carried out in mineral acids, the polymer obtained is insoluble and has a much larger chain length. 
The electro-activity of polyaniline, and particularly of electrochemically synthesised polyaniline, has been widely explored in aqueous electrolytes (MacDiarmid et al 1984). While investigating the behaviour of aniline in non aqueous media it has been observed that mechanism of polymerization and subsequent fragmentation of polymerized products are different from the aqueous media. The aniline polymer so obtained is soluble in organic solvents of higher dielectric constant such as DMSO and DMF.

The identification of the polymer so obtained has been carried out with UV visible and IR spectroscopy.

\section{Experimental}

The experimental procedure consists of polymerizing $0.1 \mathrm{M}$ of aniline in acetonitrile (containing not more than $10^{-2} \mathrm{~mol} / 1$ of water) containing $1 \mathrm{~mol}$ of either $\mathrm{LiClO}_{4}$ or $\left(n \mathrm{C}_{4} \mathrm{H}_{9}\right)_{4} \mathrm{NBF}_{4}$ as supporting electrolyte, galvanostatically in an undivided cell using either $\mathrm{Pt}$ or ITO electrodes. All electrochemical measurements reported herein are carried out with SCE as a reference electrode. The chemical polymerization of aniline was carried out by the method reported earlier (Dhawan and Trivedi 1988).

\section{Results and discussion}

Experimental evidence suggests that oxidative polymerization of aniline in nonaqueous media does not stop at the dimerization stage but that further polymerization takes place. The first inflection in the current potential curve at $0.6 \mathrm{~V}$ is assigned to the proton extraction and formation of the free radical which occurs at the interface of the electrode, and can be observed as being due to the development of a light yellow colour indicating the formation of a charge transfer complex. The free radicals thus formed dimerize to give a dication which gets further polymerized at the anode to yield a high molecular weight polymer at higher potential $(\simeq 1 \cdot 2 \mathrm{~V})$. The possible reaction mechanism may be written as

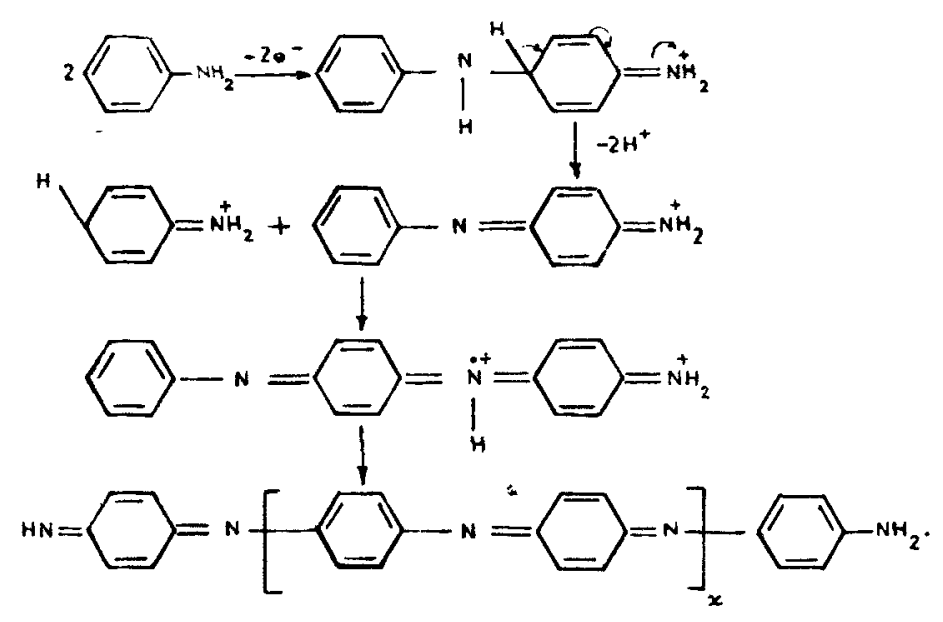


The slow evaporation of the electrolyte after removing all the insoluble polymer gave a conducting film, the conductivity of which was $1.8 \times 10^{-4} \mathrm{~s} / \mathrm{cm}$. In this film the possibility of ionic conduction due to $\mathrm{LiClO}_{4}$ or $\mathrm{TBABF}_{4}$ cannot be ruled out. An interesting observation made in the chemical polymerization of aniline in nonaqueous media is that the polymer so obtained is soluble in DMSO and DMF and less soluble in $\mathrm{CHCl}_{3}$ and $\mathrm{CH}_{3} \mathrm{OH}$. This phenomena of solubility varying with the nature of the solvent may be due to the effective solvation of the polymer matrix by solvent molecules. Further studies are in progress to understand the mechanism of solute-solvent interactions.

On comparing the UV-visible spectra of soluble polyaniline with that of the model for polyaniline proposed by $\mathrm{Lu}$ et al (1986), interesting observations were made. The phenyl capped octa aniline shows absorption bands in DMF at 332 and $618 \mathrm{~nm}$, whereas fully oxidised perinigaraniline, $\mathrm{B}_{3} \mathrm{Q}_{4}$ has absorption bands in DMF at 320 and $525 \mathrm{~nm}$. In the present studies, the polymer obtained chemically from non-aqueous media had absorption bands at $321,582 \mathrm{~nm}$ and $318,571 \mathrm{~nm}$ in DMSO and DMF respectively (figure 1). The difference in electronic spectra can be attributed to the lesser degree of conjugated polymer chains obtained in nonaqueous media than in polyaniline synthesised in aqueous media. Preliminary investigations reveal that heptamer formation occurs under these conditions.

The soluble oligomer of aniline in acetonitrile shows absorption bands at $\mathbf{3 1 0}$, 344 and $541 \mathrm{~nm}$ which are identical with the values observed for the oligomers of aniline (Yong et al 1986), when monomer units are linked at para-positions. Figure 2 shows the IR spectra of electrochemically polymerized aniline in non-aqueous media in the presence of $\mathrm{TBABF}_{4}$. The spectra is different than that of the usual spectra of polyaniline synthesized in aqueous media. The peak positions characteristic of benzenoid and quinoid ring vibration bands are shifted to 1605 and $1490 \mathrm{~cm}^{-1}$ respectively, approximately 40 and $20 \mathrm{~cm}^{-1}$ higher as compared with the usual polyaniline. Major bands observed in the IR spectrum of the

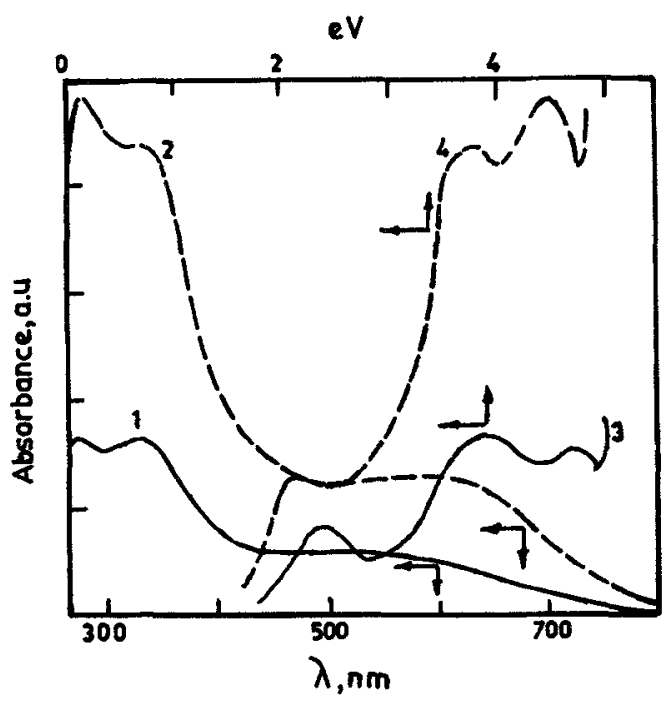

Figure 1. Electronic spectra of soluble polyaniline in DMSO (1 and 3) and DMF (2 and 4). 


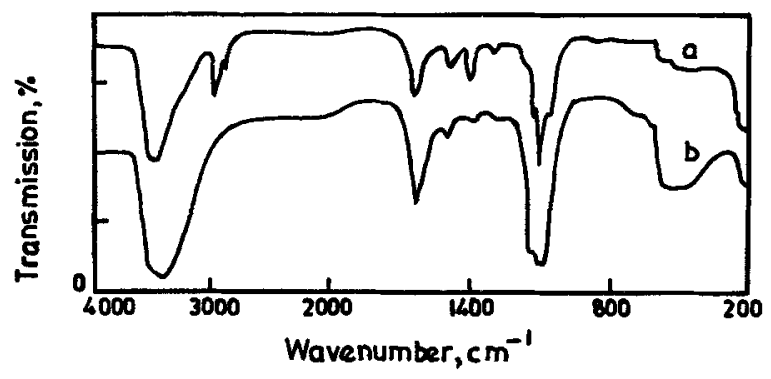

Figure 2. IR spectra of polyaniline synthesised in (a) a nonaqueous medium and (b) soluble polyaniline.

polymer obtained from non-aqueous media are (bands for the polymer from aqueous media are given in parentheses): 2940 (2917), 2860 (2854), 1605 (1565), 1490 (1470), $1300(1292)$ and $1125(1109) \mathrm{cm}^{-1}$. An additional sharp absorption band at $1080 \mathrm{~cm}^{-1}$ is observed due to $\mathrm{BF}_{4}^{-}$ion, which gets doped in the polymer during the course of the polymerization reaction.

Different opinions have been expressed regarding the polymerization of aniline under non-aqueous conditions. One group of workers (Ohsaka et al 1984) proposed that the polymerization occurs at the meta-position, while the other group (Volkov et al 1980) came to the conclusion that polymerization occurs at the para-position. Both these arguments are based only on the IR data. We are of the opinion that the polymerization occurs at the para-position and that the metaposition is not energetically suitable for the generation of free radicals which are responsible for further polymerization. Degradative analyses of the polymers and preparation of their derivatives suggest that polymerization occurs at the paraposition. The IR data also confirms this.

\section{Conclusions}

The above study reveals that short chain polymers obtained are highly soluble in DMSO and DMF and less soluble in $\mathrm{CH}_{3} \mathrm{OH}$ and $\mathrm{CHCl}_{3}$. We are of the opinion that ion-pair formation may be one of the factors responsible for the solubility of short chain polymers in these solvents. The data also reveals that the possible polymerization involves the 1,4-positions, as these positions are energetically highly suitable for obtaining stable polymers.

\section{Acknowledgement}

The authors wish to thank Prof. K I Vasu for encouragement.

\section{References}

Angelopoulos M, Ray A, MacDiarmid A G and Epstein A J 1987 Synth. Met. 2121

Bigg D M and Bradbury E J 1981 Conduct. Polym. (ed.) R B Seymour (New York: Plenum) 15 pp 23-38 
Dhawan S K and Trivedi D C 1988 Bull. Electrochem. 4729

Genies E M, Syed A A and Tsintavis C 1985 Mol. Cryst. Liq. Cryst. 121181

Green A G and Woodhead A E 1910 J. Chem. Soc. 2388

Lu F L, Wudl F, Nowak M and Heeger A J 1986 J. Am. Chem. Soc. 1088311

MacDiarmid A G, Chiang J C, Halpern M, Huang W S, Krawczyk J R, Mammone R L, Mu S L, Somasiri N L D and Wu W 1984 Polym. Prep., Am. Chem. Soc., Div. Polym. Chem. 25248

Noufi R, Nozik A J, White J and Warren L F 1982 J. Electrochem. Soc. 1292261

Ohsaka T, Ohnuki Y and Oyama N 1984 J. Electroanal. Chem. 161399

Suzhen Li, Yong C and Zhijian X 1987 Synth. Met. 20141

Volkov A, Tourillon G, Lacaze P C and Dubois J E 1980 J. Electroanal. Chem. 115279

Yong C, Suzhen Li, Zhijian X and Ding G 1986 Synth. Met. 16305 